\title{
Orthoferrite Single Crystals Growing by Modified Czochralski Method and their Properties
}

\author{
A.M. Balbashov
}

Moscow Power Engineering Institute, 111250 Moscow, Russia

\begin{abstract}
To obtain bulky large-sized orthoferrite single crystals with different rare-earth elements a new crystai growth technology is proposed including modified Czochralski method and using no metal crucibles for melt suspension. For this purpose two unusual Czochralski method modifications are used. In the first one the melt of crystallized material is obtained at heating the upper surface of polycrystalline block with intensive light radiation focused by 3 powerful single ellipsoidal mirror light concentrators, and in the second one the combination of surface heating with 3 intensive light concentrators and induction of high-frequency heating of the melt placed in cold crucible is used to produce it. In the last case the melt volume is considerably larger and this circumstance allows to obtain large-sized single crystals. For optimum thermal field formation needed for crystal pulling the relation between cold crucible diameter. frequency of inductive coil and power of the focused light inputted in the melt plays the main role. Using this technology tather perfect single crystals of yttrium. gadolinium. thulium etc. orthoferrites $25-30 \mathrm{~mm}$ in diameter and up to $50 \mathrm{~mm}$ long were grown. The maximum crystal diameter depends on the diameter of the cold crucible used and can be really increased up to 50 $\mathrm{mm}$. Yrtrium onthoferrite single crystals obtained by this technology have the following perfection characteristics: X-ray rocking curve width is $10^{\prime \prime}$ in the central part of the crystal, the dislocation density is $1-10 / \mathrm{cm}^{2}$ in the central part and up to $10^{4}$ on the periphery. blocks and twins are absent.
\end{abstract}

\section{INTRODUCTION.}

The orthoferrite single crystals $\mathrm{RFeO}_{3}$ have very interesting physical properties being promising for different technical applications, namely, high-movable magnetic domain structures, high magneto-optical figure of merit, especially in IR, very intensive magneto-resonance phenomena in millimeter wave range. However. there still remains the problem of obtaining very perfect and largely sized single crystals from these materials. The flux method of the crystal growth provides small and imperfect crystals. The Czochralski process does not work since the melt cannot hold super cooling [l]. The floating zone melting with the radiation heating permits to obtain orthoferrite single crystals of a good quality [2,3]. However, the crystal diameter is limited to about $18-20 \mathrm{~mm}$ and it is rather difficult to control the interface shape that determines the general perfection of the cristal.

We offer a new growh technology for obtaining large-sized high-quality orthoferrite single crystals, i.e. pulling the crystals from the melt holded either in "garnisage" appearing in a solid of the same composition or in a cold crucible at heating the top melt surface by the intensive light emitted by powerful xenon arc lamps and focused with the help of ellipsoidal mirror concentrators.

\section{EXPERIMENTAL PROCEDURES.}

To realize the crystal growth by pulling the Czochralski modified apparatus was designed in two variants: with radiation heating of the melt bath surface using three monoellipsoidal light concentrators arranged symmetrically around the vertical axis and with combined melt heating by the light focused on the melt surface and inductively through its volume by a high frequency . The melt in this case is held in cold crucible made of copper tubes cooled by water. The scheme of the growth apparatus for the first variant is shown in Fig.1. Ling xenon arc lamps of 5 or $10 \mathrm{~kW}$ power 3 monoellipsoidal light concentrators can appear on the surface of a polycrystalline block of raw material in its rotation ring shape heating zone. At certain light flux power value it is enough for creating the melt bath, which size and depth are determined by the light power, the degree of the light fluxes focusing. the material melting temperature and thermoconductivity. Melt super cooling zone diameter and hence crystal diameter could be changed by varying the distances of focal image points from the rotation axis. To increase the melt bath depth limiting the diameter of a grown crystal it is necessary to use an additional heating of the bottom part of a polycrystalline block up to the temperature of about $200^{\circ} \mathrm{C}$ below the material melting point. Limitations on the melt bath depth can be eliminated if the melt is placed in a cold crucible to be in addition inductively heated by a HF coil. In this case the probability to enlarge the crystal diameter essentially increases. Therewith the surface radiation heating is used, on the one hand, as a starting method of the melt production in cold crucible, when the melt received by the radiation heating is progressively related to radio frequency field (frequency equals to $1.76 \mathrm{MHz}$ ) due to a high electrical conductivity. As a result it gives a large volume of the melt. And on the other hand, it serves for forming the controlled zone of super cooling in the central part of the melt, which allows to hold the crystal pulling process. Fig. 2 gives the scheme of the crystal growth apparatus with the combined 
radiation and inductive heating. In this case the volume of the melt produced depends on a cold crucible size and HF generator power. It is really possible to get the melt volume of such materials as orthoferrites of about $1-21$ at the generator power $20-30$ $\mathrm{kW}$ and frequency 1.76-5.28 MHz. To grow large-sized crystals being more than $50 \mathrm{~mm}$ long it is necessary to feed the melt bath by a raw material during the growth process to keep the melt surface at a nearly permanent level.

For the orthoferrite crystal growth the following technological regimes were used: the pulling rate was $5-10 \mathrm{~mm} / \mathrm{h}$, the crysta! rotation speed was $60 \mathrm{r} / \mathrm{min}$., the crucible rotation speed was $5-10 \mathrm{r} / \mathrm{min}$., the crystal annealing in the growth process in a tube furnace was made at $1450^{\circ} \mathrm{C}$, the chamber was filled in with the air atmosphere. Some grown orthoferrite single crystals are shown in Fig. 3.

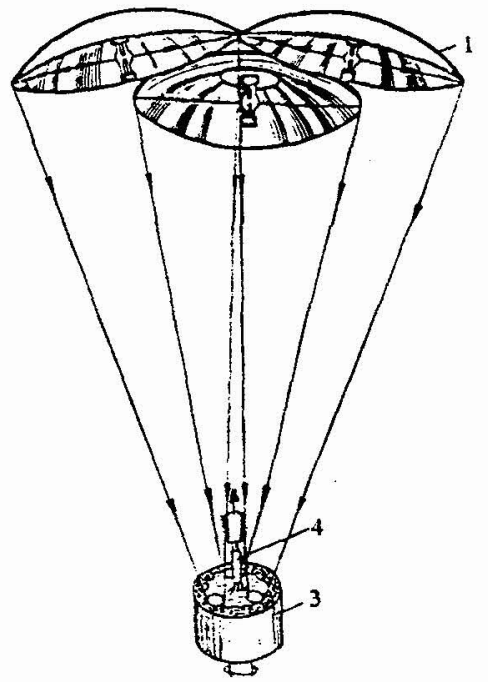

Figure 1. Principle scheme of crystal pulling equipment with radiation heating. 1 -ellipsoidal light concentrators, 3-polycrystalline block, 4-pulling crystal.

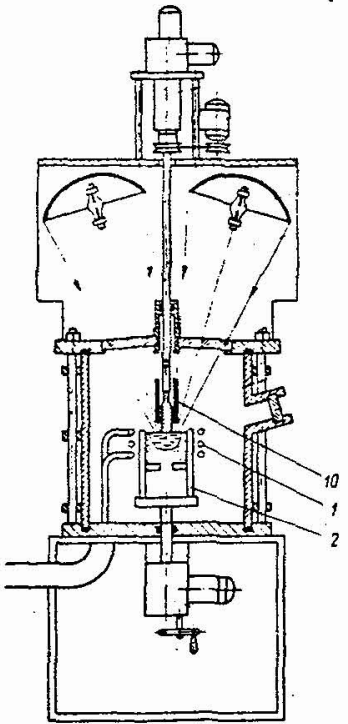

Figure 2. Apparatus for crystal pulling with combined radiation and $\mathrm{HF}$ heating.
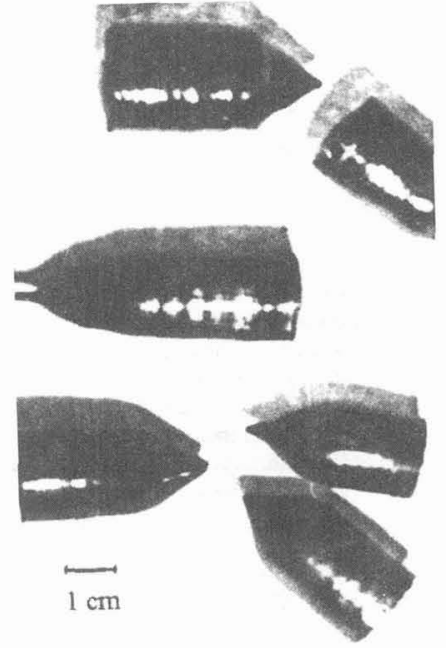

Figure 3. Orthoferrite single crystals.

10-resistive crystal annealing furnace

\section{RESULTS.}

The most important problem arising at the orthoferrite single crystal growth is to reach their high quality characterized by the absence of macro defects, such as cracks, blocks and controlled amount of micro defects: dislocations, vacancies and their clusters. The yttrium orthoferrite is the crystal mostly investigated in details because it reveals the strongest functional properties, such as domain-wall mobility, AFMR linewidth, optical absorption in visible and near infrared range.

To reach the maximum structural perfection in orthoferrite single crystals grown by the modified Czochralski method described above the crystallization interface shape control is needed, which can be realized through the careful choice of the relation between the light power absorbed by the melt and HF power transferred in the melt volume, as well as the rotation frequency of the crystal and cold crucible or polycrystalline block. For each situation, namely, for the given material, crystal diameter etc., these regime parameters are established on the base of experimentally obtained experience. As a sequence of general tendency of overheating of the central parts of the melt in a cold crucible system the fairly marked tendency to the formation of a concave crystallization interface takes place. To prevent that we recommend to hold the HF power at a minimum possible level and to work at an increased crystal rotation speed. At optimum crystal growth process regimes it is not problematic to get too close to a flat interface. In this case the crystal perfection is rather high and typically is being characterized by the following parameters (for $\mathrm{YFeO}_{3}$ crystals): the crystal diameter is $25-30 \mathrm{~mm}$, the minimum value of the X-ray rocking curve in the central part of the crystal is 10 ", the dislocation density in central part of a cross -section is $1-10 / \mathrm{cm}^{2}$ and up to $10^{4}$ on the periphery, AFMR linewidth is about 300 Oe at the room temperature, the optical absorption coefficient at the wavelength 1.5 $\mu \mathrm{m}$ is about $0.1 \mathrm{~cm}^{-1}$.

The crystal growth technology by modified Czochralski method with radiation and combined with $\mathrm{HF}$ heating were rather effective at crystal growth of another oxide materials: yttrium iron garnet, manganese zinc iron spinel and several others.

\section{References.}

[1] Daval J., Challeton D. and Mareschal J. J. Cryst. Growth 13/14(1972) 706.

[2] Okada T, Matsumi K. and Makino H. Nec. Res. Devel. 49(1970) 102-105

[3] Balbashov A.M. and Egorov S.K. J. Cryst. Growth 52(1981) 498-504 